\title{
UVA-induced upregulation of progerin suppresses 53BP1-mediated NHEJ DSB repair in human keratinocytes via progerin-lamin A complex formation
}

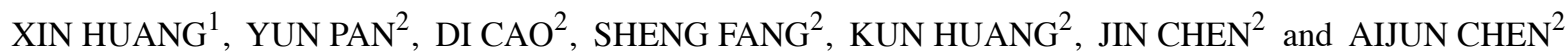 \\ ${ }^{1}$ Prescriptions Department, College of Traditional Chinese Medicine, Chongqing Medical University; \\ ${ }^{2}$ Department of Dermatology, The First Affiliated Hospital of Chongqing Medical University, Yuzhong, Chongqing, P.R. China
}

Received November 8, 2016; Accepted December 16, 2016

DOI: $10.3892 /$ or.2017.5603

\begin{abstract}
Ultraviolet (UV) radiation is the primary risk factor underlying photoaging and photocarcinogenesis. Mounting research has focused on the role of DNA damage response pathways in UV-induced double-strand break (DSB) repair. In the present study, we hypothesized that UVA-induced aberrant progerin upregulation may adversely affect p53-binding protein 1 (53BP1)-mediated non-homologous end joining (NHE) DSB repair in human keratinocytes. Basal cell carcinoma (BCC) tumors and matching normal skin tissue were sampled $(n=200)$ to investigate whether human keratinocytes display dysregulated progerin expression as a function of advancing age and BCC status. Newborn foreskin samples $(n=9)$ were used as a source for primary keratinocyte cultures. We investigated the effects of UVA radiation on progerin and lamin A expression as well as the effects of the silencing of progerin on lamin A protein expression in UVA-irradiated keratinocytes. We investigated whether blocking progerin-lamin A interaction was able to rescue UVA-induced lamin A protein downregulation, 53BP1 downregulation and 53BP1-mediated NHEJ DSB repair activity. Progerin upregulation in adult keratinocytes was associated with advancing age, not BCC status. In vitro, UVA exposure significantly upregulated progerin expression by favoring alternative LMNA gene transcript splicing. UVA exposure significantly downregulated free (unbound) lamin A protein levels via progerin-lamin A complex formation. UVA exposure significantly decreased 53BP1 protein levels via enhanced progerin-lamin A complex formation. UVA-induced progerin-lamin A complex formation was largely responsible for suppressing 53BP1-mediated NHEJ DSB repair activity. The present study is the first to demonstrate that UVA-induced progerin upregulation
\end{abstract}

Correspondence to: Professor Aijun Chen, Department of Dermatology, The First Affiliated Hospital of Chongqing Medical University, 1 YouYi Road, Yuzhong, Chongqing, P.R. China E-mail: 13062377863@163.com

Key words: UV, progerin, lamin, NHEJ, keratinocytes adversely affects 53BP1-mediated NHEJ DSB repair in human keratinocytes via progerin-lamin A complex formation.

\section{Introduction}

Ultraviolet (UV) radiation exposure is the primary risk factor underlying premature skin aging (photoaging) and skin carcinogenesis (photocarcinogenesis), with the development of cutaneous melanoma, squamous cell carcinoma (SCC), and basal cell carcinoma (BCC) all being dependent upon UV radiation exposure $(1,2)$. Unfortunately, preventive topical therapies (e.g., sunscreens and topical antioxidants) are not sufficiently effective in shielding skin cells from the DNA-damaging effects of UV radiation $(3,4)$.

Among the various DNA lesions created by UV radiation exposure, DNA double-strand breaks (DSBs) are particularly potent, since they can produce substantial losses in DNA integrity and are, therefore, more cytotoxic than other DNA lesions (5). Moreover, substandard DSB repair can result in DNA mutations or chromosomal rearrangements, which can both result in de novo carcinogenesis (5). For this reason, mounting research has focused on the role of DNA damage response pathways in DSB repair in order to develop novel chemotherapeutic targets for photoaging and photocarcinogenesis $(3,6)$.

One DNA damage response protein in particular, tumor-suppressor p53-binding protein 1 (53BP1), has been shown to play a key role in DSB repair by regulating the cellular mechanism by which DSBs are repaired (7). Specifically, 53BP1 plays a central role in the choice between 2 DSB repair pathways: homologous recombination (HR) or non-homologous end joining (NHEJ) (7). As 53BP1 inhibits an early rate-limiting step in the HR pathway and blocks recruitment of the HR pathway-promoting BRCA1 at DSBs, 53BP1 acts to promote NHEJ as the DSB repair pathway of choice $(7,8)$. As NHEJ DSB repair is the more constitutively active and efficient pathway for DSB repair, inappropriate regulation of 53BP1-mediated NHEJ can lead to genomic instability and carcinogenesis (9).

On this basis, nuclear localization of 53BP1 at UV radiation-induced foci is necessary for effective NHEJ DSB repair (10). Mechanistically, 53BP1 expression is promoted by the intermediate filament protein lamin $\mathrm{A}$, a gene product of 
the LMNA gene (7). Accordingly, lamin A deficiency (while preserving overall DNA damage response activation) results in NHEJ DSB repair inhibition from decreased nuclear 53BP1 accumulation at UV radiation-induced foci as a product of heightened 53BP1 degradation (10), while artificially reconstituting 53BP1 in the presence of lamin A deficiency has been shown to rescue NHEJ DSB repair activity (10). This evidence suggests that lamin A deficiency effectively inhibits NHEJ DSB repair activity through the decrease of the expression of 53BP1.

Progerin, an aberrant UVA radiation-induced splicing variant of the LMNA gene transcript (11), has been shown to be a potent lamin A binding partner that decreases nucleoplasmic lamin A expression (12). Notably, a recent study by Waldman et al revealed that progerin overexpression negatively impacted NHEJ DSB repair as opposed to HR DSB repair (13). On the basis of the aforementioned evidence, in the present study we hypothesized that aberrant progerin upregulation in response to UVA radiation may adversely affect 53BP1-mediated NHEJ DSB repair in human keratinocytes.

\section{Materials and methods}

Ethics statement. Approval for the present study was obtained from the Ethics Committee of the First Affiliated Hospital of Chongqing Medical University (Chongqing, China). Prior informed written consent was obtained from adult participants, and from the parents or legal guardians of the human neonatal subjects. Standards from the Guide for the Care and Use of Laboratory Animals [8th edition, National Institutes of Health (NIH), Bethesda, MD, USA] were followed for the present study.

Adult skin sampling. Study participants were recruited from outpatient clinics affiliated with the First Affiliated Hospital of Chongqing Medical University. Specifically, clinical physicians identified adult patients (aged 18-79 years) with a histologically confirmed BCC tumor diagnosed within a previous 6-month period for recruitment. Candidates were summarily excluded when they were immunocompromised, cognitively impaired, or unable to provide informed consent. BCC tumors and matching normal skin tissue samples from the recruited participants $(n=200)$ were surgically excised and stored at $-80^{\circ} \mathrm{C}$ until subsequent analysis.

Primary culture of neonatal keratinocytes. Newborn foreskin samples $(n=9)$ were used as a source of primary human neonatal keratinocytes as previously described (14). Trypsinization was performed on the proliferating primary cultures, and EpiLife culture medium containing human keratinocyte growth supplement (Life Technologies, Gaithersburg, MD, USA) was used to amplify the keratinocytes into secondary cultures as previously described (15).

Monolayer cultures were produced using passage 3 (P3) keratinocyte cultures as previously described (15). Briefly, when cells attained a $60 \%$ density, trypsinization was used to harvest the keratinocytes, and the cells were subsequently plated at 5,000 cells $/ \mathrm{cm}^{2}$. Plated cultures were incubated to develop a keratinocyte monolayer. At $50 \%$ confluence, the medium was replaced with an EpiLife ${ }^{\circledR}$-based medium free of growth factors and hormones [EpiLife ${ }^{\circledR}$ medium with only amino acids, antibiotics and hydrocortisone (Invitrogen-Cascade Biologics, Mansfield, UK)] according to the autocrine conditions. Keratinocytes were proliferated until confluence, at which point expression of the keratinocyte differentiation markers involucrin and keratin 10 were assssed.

UVA irradiation. UVA irradiation was performed as previously described (11). Irradiations were performed on culture plates resting in a temperature-regulated water bath. Growth-arrested cells were repeatedly irradiated once daily over a period of 7 days. A metal halogenide UVA lamp with an infrared radiation-specific filter (2 kW; SELLAS Sunlight, Ennepetal, Germany) was used for UVA irradiation at 5 and $10 \mathrm{~J} / \mathrm{cm}^{2}$. The emission spectrum of the lamp ranged from wavelengths of $335-440 \mathrm{~nm}$ with a peak at $375 \mathrm{~nm}$.

Lentiviral transduction. The silencing of progerin was achieved via transduction of lentiviral particles [Mission TRC short hairpin RNA (shRNA) lentiviral transduction particles (Sigma-Aldrich, St. Louis, MO, USA)] as previously described with minor modifications (15). These particles contained genes expressing resistance to puromycin and an shRNA under U6 promoter control. Two shRNAs were used: a previously described anti-progerin shRNA (16), and a non-mammalian shRNA control with no known target. Lentiviruses were used to transduce keratinocytes at a 10-fold multiplicity of infection (MOI). This was accomplished in the presence of $4 \mu \mathrm{g} / \mathrm{ml}$ of protamine sulfate (Sigma-Aldrich). At $24 \mathrm{~h}$, the culture medium was exchanged for fresh medium containing $2 \mu \mathrm{g} / \mathrm{ml}$ of puromycin (Sigma-Aldrich) for purposes of selection. When a $60 \%$ density was achieved, harvesting by trypsinization was performed, and cells were seeded in 6-well dishes for proliferation. Cells were finally evaluated for appropriate mRNA expression.

53BP1 overexpression was performed as previously described with minor modifications (17). A plasmid containing human 53BP1 cDNA was purchased from Addgene (Cambridge, MA, USA). The 53BP1 cDNA sequence was excised from the parental plasmid, and then ligated into the lentiviral pCDH1-MCS1-EF1-copGFP expression vector (System Biosciences, Mountain View, CA, USA). An empty null vector (NV) was used as a negative control. Pseudoviral particles were amplified in 293TN cells with the pPACKH1-GAG packaging plasmid, pPACKH1-REV (System Biosciences), and the pVSV-G envelope plasmid. Then, the viral particles were concentrated, and resuspended in EpiLife-based medium-free of growth factors and hormones for transduction into the cultured neonatal keratinocytes.

Quantitative reverse transcription PCR. Quantitative reverse transcription PCR (qRT-PCR) was performed as previously described with minor modifications (15). A Total RNA Isolation kit (Tiangen, Beijing, China) was used to extract total RNA, and the concentration was quantified with a NanoDrop 1000 UV/Vis spectrophotometer(ThermoScientific, Wilmington, DE, USA). SuperScript III RNase H reverse transcriptase (Invitrogen, Carlsbad, CA, USA) was applied to reverse-transcribe RNA into cDNA. The resulting cDNA was prepped with FastStart Universal SYBR-Green Master (Roche, Basel, Switzerland) as well as sense and antisense primers 

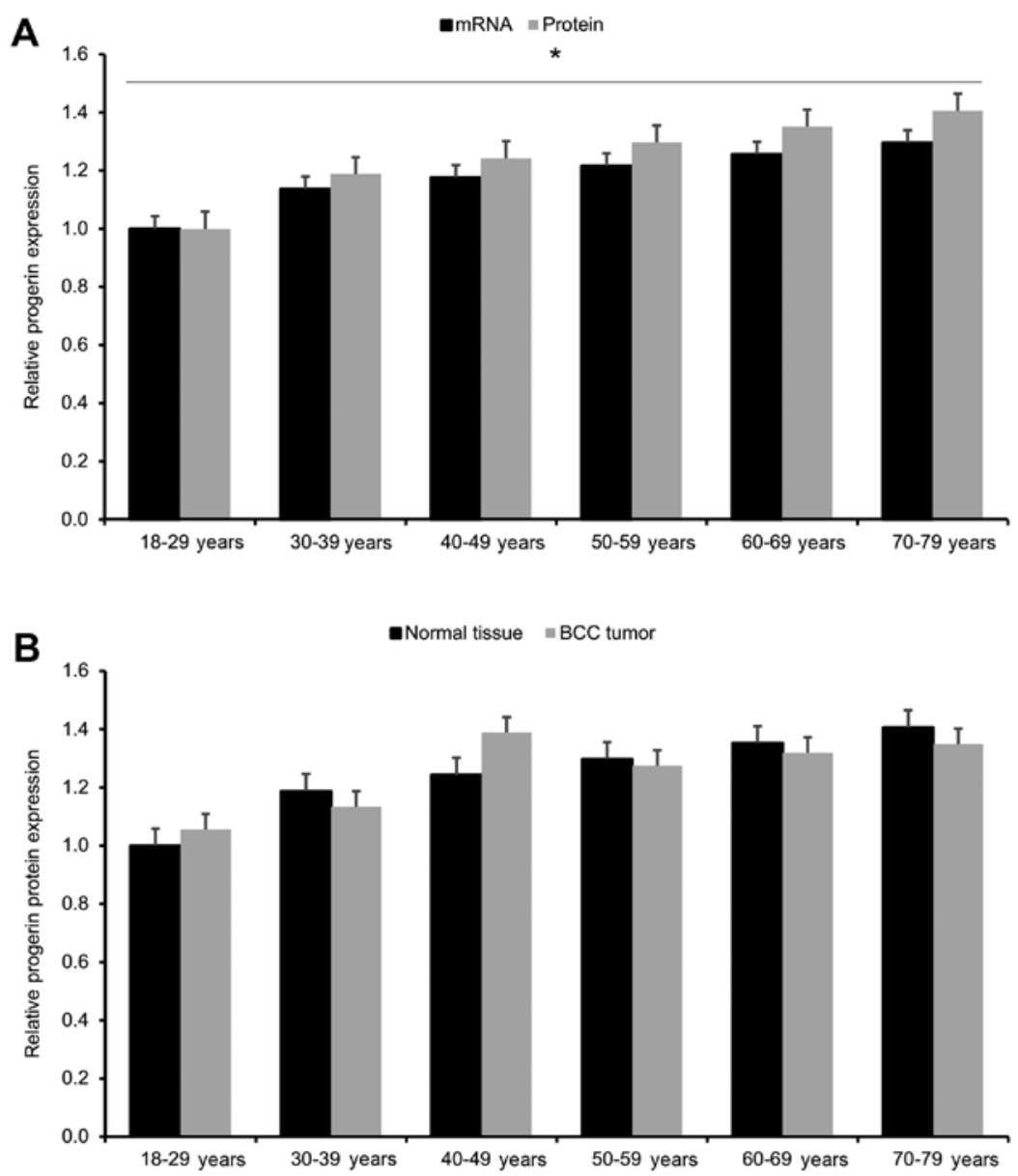

Figure 1. Human keratinocyte progerin expression correlates with advancing age, but not BCC status. Quantitative RT-PCR and western blotting were applied to assess progerin (A) mRNA and (B) protein expression, respectively. (A) Graph of progerin expression across various age groups. (B) No significant differences were observed in the protein expression of progerin between BCC tumors and matched normal tissues across all age groups; $\beta$-actin was employed as a loading control. Data are displayed as the means \pm SEMs; ${ }^{*} \mathrm{p}<0.05$. BCC, basal cell carcinoma.

(300 nM; Sigma-Aldrich), which then underwent amplification on an ABI 7300 Real-Time PCR System (Applied Biosystems, Foster City, CA, USA). The following primers were applied: lamin A forward, 5'-TCTTCTGCCTCCAGTGTCACG-3' and reverse, 5'-AGTTCTGGGGGCTCTGGGT-3'; progerin forward, 5'-ACTGCAGCAGCTCGGGG-3' and reverse 5'-GGCTCTGGGCTCCTGAGCC-3'; 53BP1 forward, 5'-GCC TGATCAATGGACCCTACTGGAAGTCAGG-3', and reverse, 5'-CCGCTCGAGTTAGTGAGAAACATAATCGTG TTT-3'; and $\beta$-actin forward, 5'-GGAGACAAGCTTGCTCA TCACCATTGGCAATGAGCG-3', and reverse, 5'-GCGAAT TCGAGCTCTAGAAGCATTTGCGGTGGACG-3'. $\beta$-actin expression levels were used in the normalization of the target mRNAs.

Western blotting. Western blotting was performed as previously described with minor modifications (15). Lysis buffer (consisting of $62.5 \mathrm{mM}$ Tris- $\mathrm{HCl}, 8.7 \%$ glycerol, $2 \%$ SDS and $0.2 \%$ dithiothreitol; Sigma-Aldrich) was used to extract proteins from keratinocytes. The Pierce $660 \mathrm{~nm}$ Protein Assay Reagent (cat. no. 22660) in conjunction with anti-SDS (Thermo Scientific) was used to assess protein concentrations. The separation of proteins $(20 \mu \mathrm{g})$ was performed with $10 \%$ SDS-PAGE and then the proteins were transferred to polyvinylidene fluoride (PVDF) membranes. A solution of 5\% powdered milk diluted in PBS and $0.1 \%$ Tween-20 was used to saturate the membranes. The membranes were then incubated for $1 \mathrm{~h}$ with the following primary antibodies (all diluted 1:1,000): anti-lamin A, anti-lamin A/C (both from Santa Cruz Biotechnology, Santa Cruz, CA, USA), anti-progerin, anti-53BP1 and anti- $\beta$-actin (all from Abcam, Cambridge, MA, USA). The washed membranes were incubated for $1 \mathrm{~h}$ with the appropriate HRP-conjugated secondary antibodies (diluted 1:5,000; Boster Company, Wuhan, China). The ECL Western Blotting Detection kit (Applygen, Beijing, China) was used for chemiluminescent signal detection.

Progerin-lamin A interaction assay. Direct interaction of progerin and lamin A in cultured neonatal keratinocytes was evaluated as previously described (12). Briefly, streptavidin-bead precleared cell lysates were sequentially incubated with biotin-JH4 or biotin and streptavidin beads. Then, western blotting was performed on the precipitated materials. To block the interaction of progerin and lamin A, a 24-h treatment with JH4 $(5 \mu \mathrm{M})$ was performed. The farnesyltransferase inhibitor FTI-277 [which does not affect progerin-lamin A binding (12)] was applied as a negative control for JH4. Cell lysates were subjected to immunoprecipitation (IP) with a lamin A-specific antibody (Santa Cruz Biotechnology) followed by immunoblotting with a progerin-specific antibody (Abcam). A cell line 

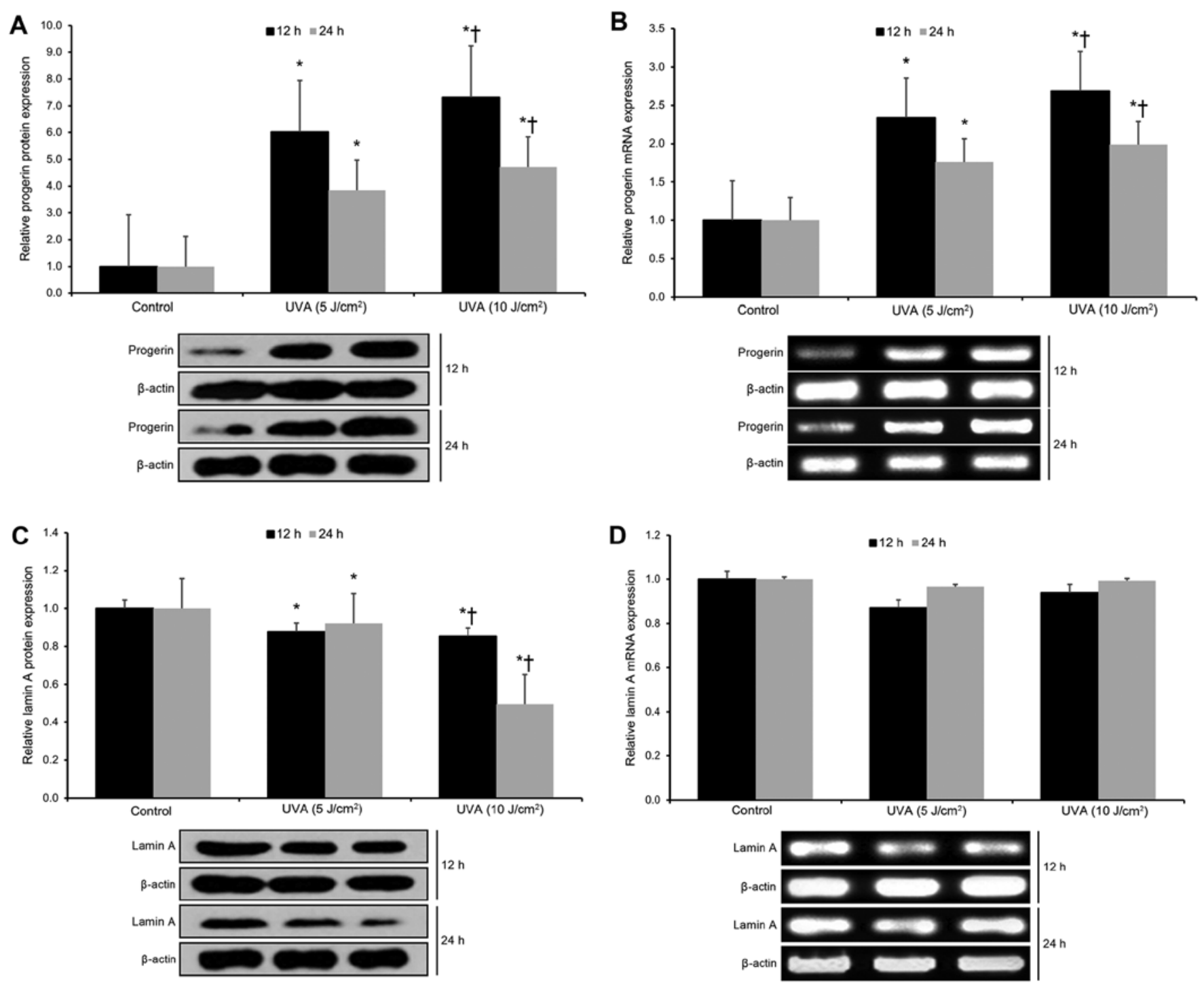

Figure 2. UVA induces progerin upregulation in human keratinocytes but has no affect on lamin A transcription. Graphs of (A) progerin protein expression, (B) progerin mRNA expression, (C) lamin A protein expression and (D) lamin A mRNA expression following UVA exposure (5 or $\left.10 \mathrm{~J} / \mathrm{cm}^{2}\right)$ assessed at 12 or $24 \mathrm{~h}$ post-irradiation; $\beta$-actin was employed as a loading control. Data are displayed as the means \pm SEMs; ${ }^{*} \mathrm{p}<0.05$ compared to the control group, ${ }^{\dagger} \mathrm{p}<0.05$ compared to the $5 \mathrm{~J} / \mathrm{cm}^{2}$ group.

of untreated neonatal keratinocytes was applied as a negative control for progerin expression.

Neutral comet assays. The CometSlide assay kit (Trevigen, Gaithersburg, MD, USA) was used in neutral comet assay procedures as previously described with minor modifications (10). Irradiation doses were set at $10 \mathrm{~J} / \mathrm{cm}^{2}$ at an incubation temperature of $37^{\circ} \mathrm{C}$. Incubation times were varied $(0,30,60,90,120$ and $150 \mathrm{~min})$ to allow for the repair of DNA damage. Agarose was used to embed the keratinocytes, which were then lysed and treated with neutral electrophoresis. Previous to image analysis, the keratinocytes underwent tethidium bromide staining and were examined using fluorescence microscopy. The result of single-cell electrophoresis was a comet-shaped DNA distribution; an intact and high molecular weight DNA constitute the comet head, while the tail consists of leading ends of migrating fragments. The olive comet moment (OCM) was determined by multiplying the percentage of tail DNA by the displacement between the head and tail distribution means (10). The OCM was calculated via CometScore ${ }^{\mathrm{TM}}$ version 1.5 (TriTek Corp., Sumerduck, VA, USA). A total of 25-30 comets/sample were analyzed in each experiment.

\section{Results}

In the present study, we first investigated whether human keratinocytes display dysregulated progerin expression as a function of advancing age and BCC status. We found that progerin expression in human keratinocytes gradually increased with advancing age ( $\mathrm{p}<0.05$; Fig. 1A). However, upon comparing each age group, we found no significant differences in progerin protein expression between BCC tumors and matched normal tissues across all age groups ( $<<0.05$; Fig. 1B). These findings indicate that progerin upregulation in human keratinocytes is associated with advancing age, not BCC status.

Next, we investigated the effects of UVA radiation on progerin expression in cultured human neonatal keratinocytes. 

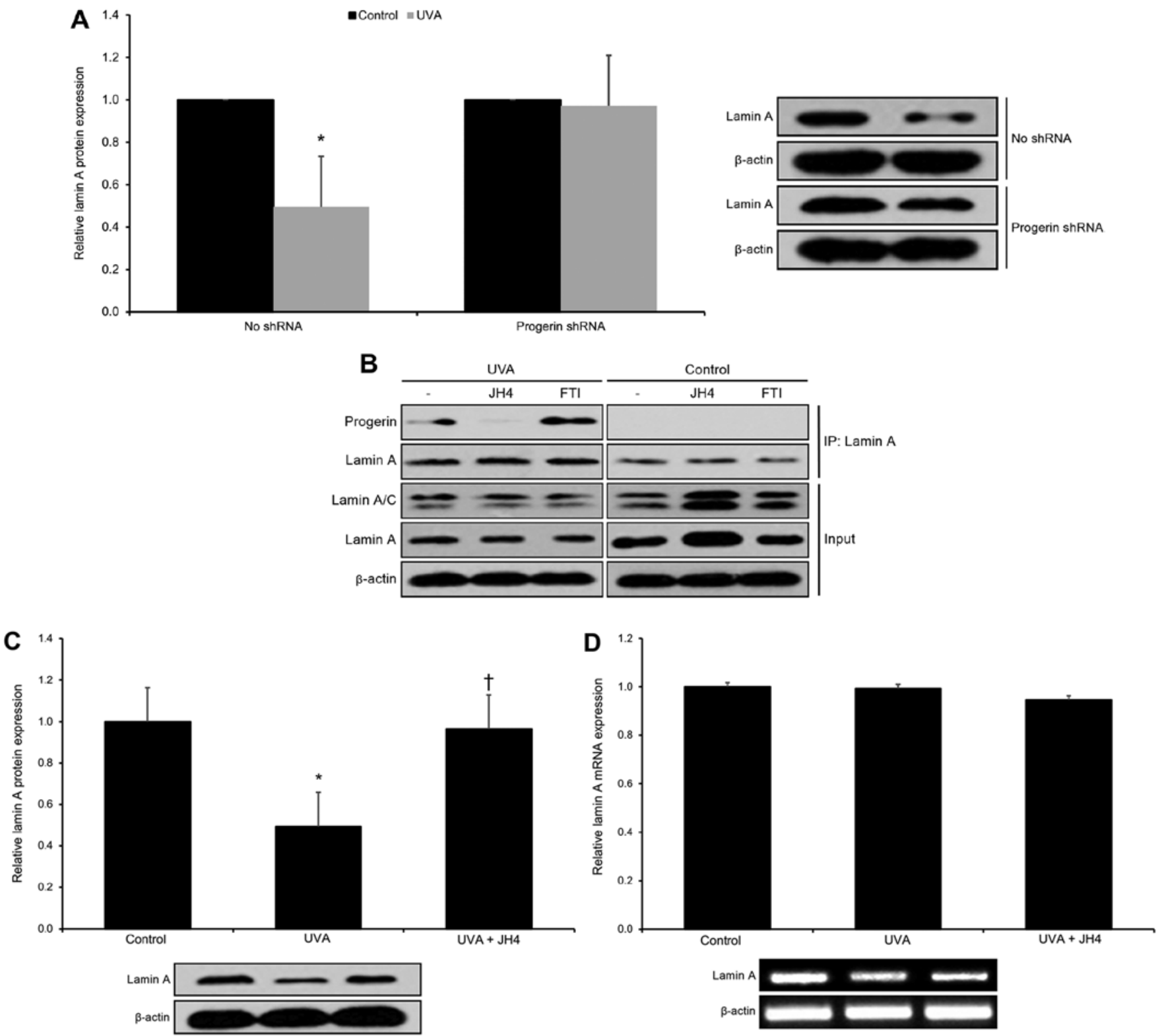

Figure 3. UVA-induced progerin upregulation decreases wild-type lamin A expression through progerin-lamin A complex formation. (A) Graph of lamin A protein expression following UVA exposure $\left(10 \mathrm{~J} / \mathrm{cm}^{2}\right)$ assessed at $24 \mathrm{~h}$ post-irradiation. Progerin shRNA rescues lamin A protein expression in UVA-treated cells. (B) JH4 blocks progerin-lamin A binding in keratinocytes. A 24-h treatment period of JH4 $(5 \mu \mathrm{M})$ (but not a control compound FTI-277) blocked progerin-lamin A binding in kertainocytes. Whole cell lysates were immunoprecipitated (IP) with a lamin A-specific antibody, and then immunoblotted with a progerin-specific antibody. Untreated normal keratinocytes were employed as a negative control for progerin expression. (C) Blocking of progerin-lamin A binding by JH4 rescues lamin A protein expression. (D) However, no effect upon lamin A transcription was observed. Cells were treated with JH4 for $24 \mathrm{~h}$ prior to immunoblotting and quantitative RT-PCR; $\beta$-actin was employed as a loading control. Data are displayed as the means \pm SEMs; " $p<0.05$ compared to the control group, ${ }^{\dagger} \mathrm{p}<0.05$ compared to the UVA group.

We found that UVA exposure (at both 5 and $10 \mathrm{~J} / \mathrm{cm}^{2}$ doses) resulted in significant upregulation of progerin protein expression at 12 and $24 \mathrm{~h}$ post-irradiation (p<0.05; Fig. 2A). Moreover, UVA exposure (at both 5 and $10 \mathrm{~J} / \mathrm{cm}^{2}$ doses) resulted in significant upregulation of progerin mRNA expression at 12 and 24 h post-irradiation ( $<<0.05$; Fig. 2B). These findings indicate that UVA exposure significantly upregulates progerin expression in human keratinocytes by favoring alternative LMNA gene transcript splicing.

Next, we investigated the effects of UVA radiation on lamin A expression in cultured human neonatal keratinocytes. We found that UVA exposure (at both 5 and $10 \mathrm{~J} / \mathrm{cm}^{2}$ doses) resulted in significant downregulation of free (unbound) lamin A protein expression at 12 and $24 \mathrm{~h}$ post-irradiation ( $<<0.05$; Fig. 2C). However, UVA exposure (at both 5 and $10 \mathrm{~J} / \mathrm{cm}^{2}$ doses) did not significantly affect lamin A mRNA expression at 12 and $24 \mathrm{~h}$ post-irradiation ( $p>0.05$; Fig. 2D). These findings indicate that UVA exposure significantly downregulates lamin A expression in human keratinocytes by decreasing free lamin A protein levels (as opposed to downregulating lamin A transcription).

In order to further investigate the mechanism by which UVA exposure decreases free lamin A protein levels in human keratinocytes, we investigated the effects of silencing progerin on free lamin A protein expression in UVA-irradiated cultured human neonatal keratinocytes. We found that silencing of 

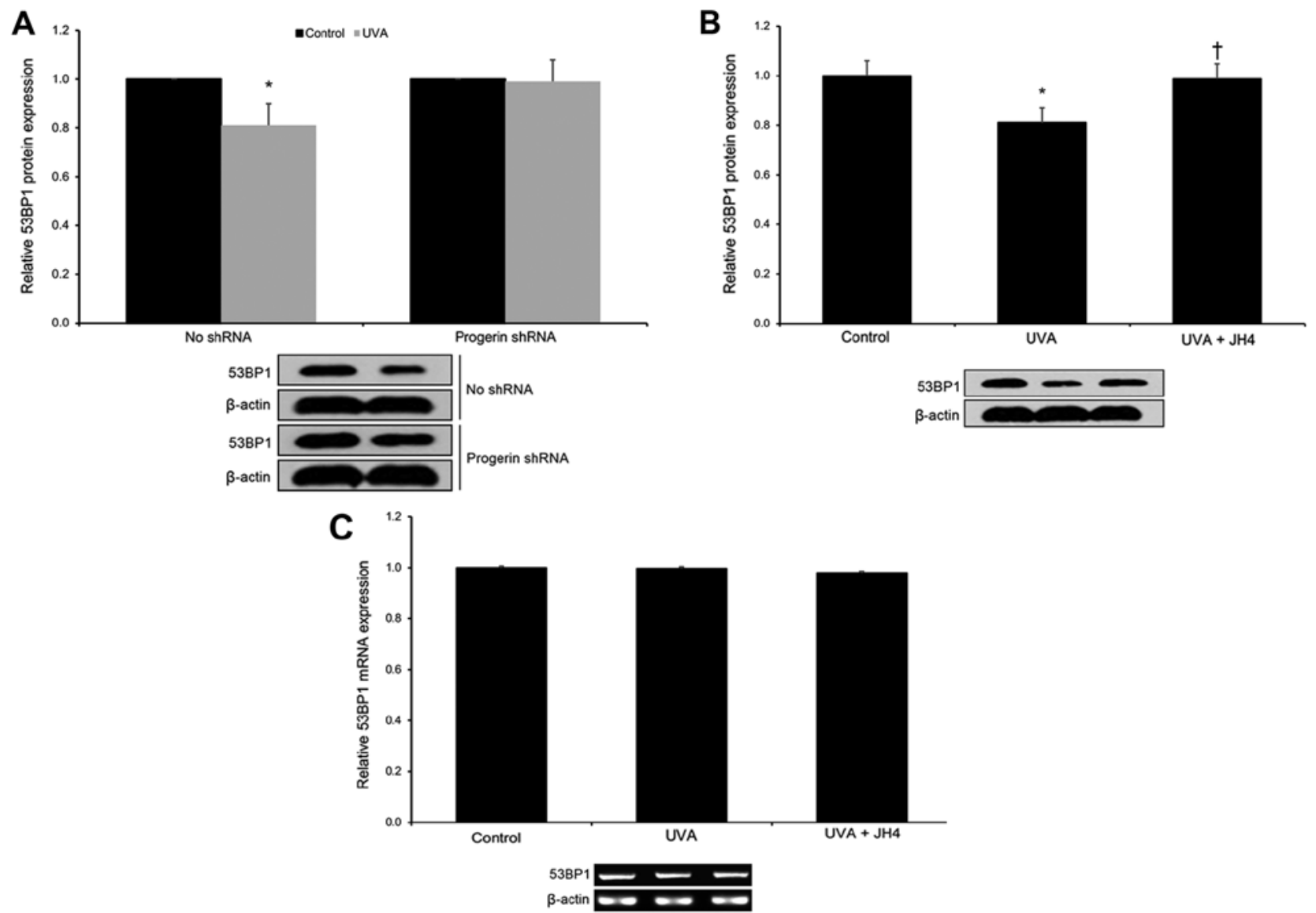

Figure 4. UVA-induced progerin upregulation decreases 53BP1 expression through progerin-lamin A complex formation. (A) Graph of 53BP1 protein expression following UVA exposure $\left(10 \mathrm{~J} / \mathrm{cm}^{2}\right)$ assessed at $24 \mathrm{~h}$ post-irradiation. Progerin shRNA rescues 53BP1 protein expression in UVA-treated cells. (B) Blocking of progerin-lamin A binding by JH4 rescues 53BP1 protein expression. (C) However, no effect upon 53BP1 transcription was observed. Cells were treated with $\mathrm{JH} 4$ for $24 \mathrm{~h}$ prior to immunoblotting and quantitative RT-PCR. $\beta$-actin was employed as a loading control. Data are displayed as the means \pm SEMs; ${ }^{*} \mathrm{p}<0.05$ compared to the control group, ${ }^{\dagger} \mathrm{p}<0.05$ compared to the UVA group.

progerin (via a progerin-specific shRNA) was able to rescue UVA-induced free lamin A protein downregulation $(\mathrm{p}<0.05$; Fig. 3A). From these data, we hypothesized that progerin-lamin A interaction may result in the observed downregulation in free lamin A protein levels in UVA-irradiated keratinocytes. In order to test this hypothesis, we selected the chemical compound JH4, which has been previously shown to prevent progerin-lamin A binding in HGPS cells (12). We then ascertained that $\mathrm{JH} 4$ blocked the interaction of progerin and lamin A in cultured human keratinocytes (Fig. 3B). Next, we demonstrated that blocking progerin-lamin A interaction by $\mathrm{JH} 4$ was able to rescue UVA-induced lamin A protein downregulation ( $\mathrm{p}<0.05$; Fig. 3C). As a control experiment, we ascertained that $\mathrm{JH} 4$ administration produced no significant effects on lamin A mRNA levels ( $p>0.05$; Fig. 3D). These findings indicate that UVA exposure significantly decreases free lamin A protein levels in human keratinocytes through the promotion of progerin-lamin A complex formation.

As lamin A has been shown to promote 53BP1 levels (7), we next hypothesized that UVA-induced progerin-lamin A complex formation (via the decrease of free lamin A protein levels) may result in 53BP1 downregulation in UVA-irradiated cultured human neonatal keratinocytes. First, we found that silencing progerin (via a progerin-specific shRNA) was able to rescue UVA-induced 53BP1 protein downregulation ( $\mathrm{p}<0.05$; Fig. 4A). Next, we demonstrated that blocking progerin-lamin A interaction by $\mathrm{JH} 4$ was able to rescue UVA-induced 53BP1 protein downregulation $(\mathrm{p}<0.05$; Fig. 4B). As a control experiment, we ascertained that JH4 administration produced no significant effects on 53BP1 mRNA levels ( $p>0.05$; Fig. 4C). These findings indicate that UVA exposure significantly decreases 53BP1 protein levels in human keratinocytes via enhanced progerin-lamin A complex formation.

As lamin A deficiency has been shown to inhibit NHEJ DSB repair through the promotion of 53BP1 degradation (10), we next hypothesized that UVA-induced progerin-lamin A complex formation may inhibit NHEJ DSB repair in UVA-irradiated cultured human neonatal keratinocytes. In order to test this hypothesis, we performed a set of neutral comet assays on cultured human neonatal keratinocytes. As expected, we found that UVA irradiation significantly decreased NHEJ DSB repair activity ( $p<0.05$; Fig. 5A). Notably, JH4 treatment was able to fully rescue NHEJ DSB repair activity irrespective of 53BP1 overexpression $(\mathrm{p}<0.05$; Fig. 5A). However, 53BP1 overexpression alone was only able to partially rescue NHEJ DSB repair activity ( $<<0.05$; Fig. 5A). These findings indicate that UVA-induced progerin-lamin A complex formation is largely responsible for the inhibition of 53BP1-mediated NHEJ DSB repair activity. 

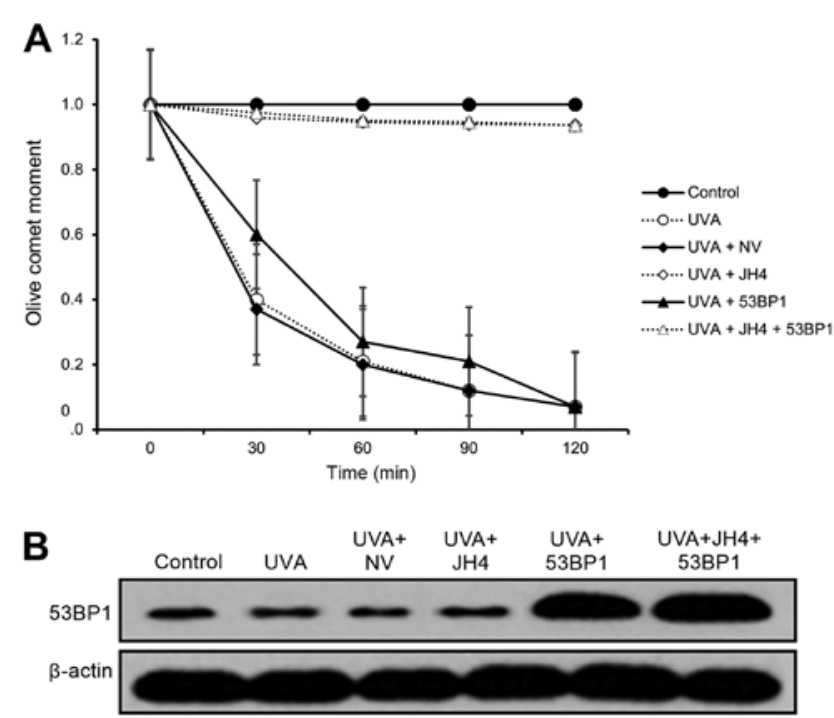

Figure 5. UVA-induced progerin-lamin A complex formation inhibits 53BP1-mediated NHEJ DSB repair. (A) Blocking progerin-lamin A binding by JH4 rescued classical NHEJ in a 53BP1-dependent manner. Keratinocytes were treated with $\mathrm{JH} 4$ for $24 \mathrm{~h}$ prior to immunoblotting. Keratinocytes were UVA-irradiated at $10 \mathrm{~J} / \mathrm{cm}^{2}$. At various post-irradiation time-points $(0,30,60$, 90 and $120 \mathrm{~min}$ ), neutral comet assays were performed on the keratinocytes. The olive comet moment (a quantitative measure of unrepaired DNA) was assessed to determine repair activity of IR-induced double-strand breaks Approximately 60 cells were analyzed/sample/time-point. (B) Immunoblots confirming 53BP1 protein overexpression in keratinocytes transduced with a 53BP1 overexpression vector. An empty null vector (NV) was applied as a transduction control; $\beta$-actin was employed as a loading control. Data are displayed as the means \pm SEMs. NHEJ, non-homologous end joining; DSB, double-strand break.

\section{Discussion}

Progerin is a shortened, farnesylated splicing variant of the LMNA gene, which has been most popularly associated with the accelerated aging genetic disorder termed Hutchinson-Gilford progeria syndrome (HGPS) (18). The progerin protein dysfunctionally localizes on the inner surface of the nuclear membrane, where it interferes with several critical lamin A-associated nuclear processes such as chromatin organization, DNA damage response pathways, gene transcription activity, and maintaining telomere integrity (19). Notably, progerin is expressed at low levels in healthy people and increases in expression over the course of normal physiological aging (20); moreover, progerin upregulation has also been associated with prostate cancer and renal cell carcinoma (21). Thus, an improved understanding of the role of progerin in aging and carcinogenesis may be helpful in developing anti-aging and anticancer therapeutics. Unfortunately, there has been limited research regarding the role of progerin in photoaging and photocarcinogenesis in skin keratinocytes. The present study is the first to demonstrate that UVA-induced progerin upregulation adversely affects 53BP1-mediated NHEJ DSB repair in human keratinocytes via progerin-lamin A complex formation.

First, from a large population of human skin samples, we discovered that progerin upregulation in human keratinocytes is significantly associated with advancing age, not BCC status. This finding concurs with a study by Miller et al which showed increased fibroblastic progerin expression in aged human patients and demonstrated that experimentally induced progerin expression in induced pluripotent stem cell (iPSC)-derived fibroblasts and neurons induces several aging-related characteristics in vitro (19). Our findings also concur with those of Olive et al which discovered progerin expression in coronary arteries of aged human patients and demonstrated that coronary arterial progerin expression significantly increases with advancing age (22). Second, we found that UVA exposure significantly upregulates progerin expression in human keratinocytes by favoring alternative LMNA gene transcript splicing. These findings concur with those of the Firoozan et al and Takeuchi et al research groups, which have shown that UVA radiation induces progerin upregulation in human melanocytes and fibroblasts $(11,23)$. Moreover, they also demonstrated that this UVA-induced progerin upregulation is mediated by radiation-driven oxidative damage, which promotes alternative splicing of the LMNA gene transcript (11). As these combined findings show an association between age, UVA exposure and progerin upregulation in human keratinocytes, but no significant association between BCC status and progerin expression, our findings suggest that progerin upregulation in human keratinocytes is associated with photoaging, but not photocarcinogenesis.

A recently published study by Lee et al revealed that lamin A is an important binding target of progerin (12). Moreover, they also demonstrated that the chemical compound JH4 selectively binds to progerin, thereby blocking progerin-lamin A complex formation (12). Therefore, we applied JH4 in order to determine whether the effects of UVA on lamin A protein levels are affected by progerin-lamin A complex formation. First, we found that the silencing of progerin was able to rescue UVA-induced free lamin A protein downregulation. Second, after ascertaining that JH4 blocks the interaction of progerin and lamin A in cultured human keratinocytes, we found that JH4 treatment was able to rescue free lamin A protein expression in UVA-irradiated keratinocytes. These findings demonstrate that UVA exposure significantly decreases free lamin A protein levels in human keratinocytes through the promotion of progerin-lamin A complex formation.

Lamin A deficiency has been shown to decrease nuclear accumulation of the DNA damage response protein 53BP1 at UV-induced foci through the enhancement of 53BP1 degradation (10). In the present study, we demonstrated that UVA exposure significantly decreased 53BP1 protein levels in human keratinocytes via enhanced progerinlamin A complex formation (a process which decreased free lamin A levels). However, we did not examine the precise mechanism(s) by which the complex formation-driven decrease in free lamin A levels affects 53BP1 protein expression, as these have been reported elsewhere. A previous study by Gonzalez-Suarez et al, demonstrated that lamin A deficiency drives cysteine protease cathepsin L (CTSL)-mediated degradation of the 53BP1 protein (24). Moreover, Gibbs-Seymour et al demonstrated that lamin A directly binds to the 53BP1 Tudor domain via a ataxia telangiectasia mutated (ATM)-mediated mechanism, thereby promoting nuclear 53BP1 retention (7). As 53BP1 is a key effector of lamin A (7), further research on the downstream 
mechanism(s) involved in the effects of free lamin A on 53BP1 protein degradation and nuclear localization are still needed.

Progerin has been shown to slow NHEJ DSB DNA repair, which leads to heightened DSB accumulation over time (13). Specifically, progerin-overexpressing cells display a significantly increased DSB event frequency via NHEJ, with no significant effect observed upon DSB event frequency via HR (13). Notably, in the present study, JH4 treatment was able to fully rescue NHEJ DSB repair activity irrespective of 53BP1 overexpression, while 8-fold 53BP1 overexpression alone was only able to partially rescue NHEJ DSB repair activity. These combined findings suggest that UVA-induced progerin-lamin A complex formation is primarily responsible for 53BP1-mediated losses in NHEJ DSB repair activity, but there are other non-53BP1-mediated mechanism(s) involved in NHEJ DSB repair activity that are also adversely affected by progerin-lamin A complex formation. Indeed, Ghosh et al have reported that free lamin A protein recruits and facilitates sirtuin 6 (SIRT6)-mediated NHEJ DSB repair $(25,26)$. Thus, UVA-induced progerin-lamin A complex formation may adversely affect NHEJ DSB repair through the attenuation of both 53BP1 and SIRT6 pathways. As this field of investigation is relatively novel, further studies are still needed to examine the downstream effector(s) of lamin A that affect DSB repair activity.

In conclusion, the present study is the first to demonstrate that UVA-induced progerin upregulation adversely affects 53BP1-mediated NHEJ DSB repair in human keratinocytes via progerin-lamin A complex formation. These findings improve our understanding of the role of progerin in photoaging and may provide additional insights to support the development of anti-aging skin therapeutics.

\section{Acknowledgements}

The present study was supported by the Natural Science Foundation of China (81573027).

\section{References}

1. Moan J, Grigalavicius M, Baturaite Z, Dahlback A and Juzeniene A: The relationship between UV exposure and incidence of skin cancer. Photodermatol Photoimmunol Photomed 31: 26-35, 2015.

2. Iannacone MR, Hughes MCB and Green AC: Effects of sunscreen on skin cancer and photoaging. Photodermatol Photoimmunol Photomed 30: 55-61, 2014.

3. Kabir Y, Seidel R, Mcknight B and Moy R: DNA repair enzymes: An important role in skin cancer prevention and reversal of photodamage - a review of the literature. J Drugs Dermatol 14: 297-303, 2015.

4. Ferlay J, Soerjomataram I, Dikshit R, Eser S, Mathers C, Rebelo M, Parkin DM, Forman D and Bray F: Cancer incidence and mortality worldwide: Sources, methods and major patterns in GLOBOCAN 2012. Int J Cancer 136: E359-E386, 2015.

5. Schwertman P, Bekker-Jensen S and Mailand N: Regulation of DNA double-strand break repair by ubiquitin and ubiquitin-like modifiers. Nat Rev Mol Cell Biol 17: 379-394, 2016.

6. Surovtseva Y, Jairam V, Sundaram R, Bindra R and Herzon S: A high-throughput, high-content assay for the discovery of new inhibitors of DNA double-strand break repair. Cancer Res 76 (Suppl 14): 2174, 2016. doi: 10.1158/1538-7445.AM2016-2174
7. Gibbs-Seymour I, Markiewicz E, Bekker-Jensen S, Mailand N and Hutchison CJ: Lamin A/C-dependent interaction with 53BP1 promotes cellular responses to DNA damage. Aging Cell 14: 162-169, 2015.

8. Canny M, Wan L, Fradet-Turcotte A, Orthwein A, Moatti N, Juang YC, Zhang W, Noordermeer SM, Wilson MD, Vorobyov A, et al: A genetically encoded inhibitor of 53BP1 to 1 stimulate homology-based gene editing. bioRxiv: 060954, 2016. doi: https://doi.org/10.1101/060954.

9. Her SC and Her C: Targeting DNA double-strand break repair in cancer therapy. J Mol Genet Med 9: e106, 2015.

10. Redwood AB, Perkins SM, Vanderwaal RP, Feng Z, Biehl KJ, Gonzalez-Suarez I, Morgado-Palacin L, Shi W, Sage J, Roti-Roti JL, et al: A dual role for A-type lamins in DNA double-strand break repair. Cell Cycle 10: 2549-2560, 2011.

11. Takeuchi H and Rünger TM: Longwave UV light induces the aging-associated progerin. J Invest Dermatol 133: 1857-1862, 2013.

12. Lee SJ, Jung YS, Yoon MH, Kang SM, Oh AY, Lee JH, Jun SY, Woo TG, Chun HY, Kim SK, et al: Interruption of progerin-lamin $\mathrm{A} / \mathrm{C}$ binding ameliorates Hutchinson-Gilford progeria syndrome phenotype. J Clin Invest 126: 3879-3893, 2016.

13. Waldman AS, Chowdhary S, Patrick A, Hersey $M$ and Waldman BC: The influence of progerin expression on the nature of DNA double-strand break repair. The FASEB J 30: (Suppl 1): S576.4, 2016

14. Mistry DS, Chen Y, Wang Y and Sen GL: Transcriptional profiling of SNAI2 regulated genes in primary human keratinocytes. Genom Data 4: 43-46, 2015.

15. Malaisse J, Pendaries V, Hontoir F, De Glas V, Van Vlaender D, Simon M, Lambert de Rouvroit C, Poumay Y and Flamion B: Hyaluronan does not regulate human epidermal keratinocyte proliferation and differentiation. J Biol Chem 291: 6347-6358, 2016.

16. Zhang H, Xiong Z-M and Cao K: Mechanisms controlling the smooth muscle cell death in progeria via down-regulation of poly(ADP-ribose) polymerase 1. Proc Natl Acad Sci USA 111: E2261-E2270, 2014

17. Woods M, Pant R and Mallya SM: Cyclin D1 and cyclin D-dependent kinases enhance oral keratinocyte proliferation but do not block keratinocyte differentiation. Int $\mathbf{J}$ Oncol 37: 1471-1475, 2010.

18. Musich PR and Zou Y: DNA-damage accumulation and replicative arrest in Hutchinson-Gilford progeria syndrome. Biochem Soc Trans 39: 1764-1769, 2011.

19. Miller JD, Ganat YM, Kishinevsky S, Bowman RL, Liu B, Tu EY, Mandal PK, Vera E, Shim JW, Kriks S, et al: Human iPSC-based modeling of late-onset disease via progerin-induced aging. Cell Stem Cell 13: 691-705, 2013.

20. Kubben N, Zhang W, Wang L, Voss TC, Yang J, Qu J, Liu GH and Misteli T: Repression of the antioxidant NRF2 pathway in premature aging. Cell 165: 1361-1374, 2016.

21. Kennedy BK: A new connection between VHL and cancer threads through progerin. Cell Cycle 12: 2721-2722, 2013.

22. Olive M, Harten I, Mitchell R, Beers JK, Djabali K, Cao K, Erdos MR, Blair C, Funke B, Smoot L, et al: Cardiovascular pathology in Hutchinson-Gilford progeria: Correlation with the vascular pathology of aging. Arterioscler Thromb Vasc Biol 30: 2301-2309, 2010.

23. Firoozan S, Takeuchi $\mathrm{H}$ and Ruenger T: Longwave ultraviolet light induces the aging-associated progerin in melanocytes. Am J Med Genet 2603: 2624, 2006.

24. Gonzalez-Suarez I, Redwood AB, Grotsky DA, Neumann MA, Cheng EHY, Stewart CL, Dusso A and Gonzalo S: A new pathway that regulates 53BP1 stability implicates cathepsin L and vitamin D in DNA repair. EMBO J 30: 3383-3396, 2011.

25. Ghosh S, Liu B, Wang Y, Hao Q and Zhou Z: Lamin A is an endogenous SIRT6 activator and promotes SIRT6-mediated DNA repair. Cell Reports 13: 1396-1406, 2015.

26. Mao Z, Hine C, Tian X, Van Meter M, Au M, Vaidya A, Seluanov A and Gorbunova V: SIRT6 promotes DNA repair under stress by activating PARP1. Science 332: 1443-1446, 2011. 